\title{
Dermatomyositis Presented with Facial Rash in a 40 Years Old Lady in Bangladesh: A Case Report
}

\author{
Meera Momtaz Sabeka1, Mohammad Enayet Hussain² \\ ${ }^{1}$ Consultant Neurologist, William Harvey Hospital, Kent, UK; ${ }^{2}$ Associate Professor, Department of Neurology, \\ National Institute of Neurosciences \& Hospital, Dhaka, Bangladesh
}

[Received: 30 March 2019 Accepted: 2 May 2019; Published: 1 July 2019]

\begin{abstract}
Dermatomyositis is overly simplified as inflammatory myopathy with characteristic rashes. Understanding dermatomyositis is challenging because firstly presentation may be non-specific, vague and delayed. Secondly there is no specific diagnostic test or universally acceptable diagnostic criteria. Therefore, to missed or delayed diagnosis results in failure to treatment. A forty year old lady with facial rash and generalized weakness was diagnosed with dermatomyositis on the basis of generalized weakness, typical facial rash with very high CK and eletrophysiological study. Steroid treatment initially improved her symptoms. [Journal of National Institute of Neurosciences Bangladesh, 2019;5(2): 191-193]
\end{abstract}

Keywords: Dermatomyositis; generalized weakness; facial rash

Correspondence: Dr. Meera Momtaz Sabeka, MD, MPH, MRCP. Consultant neurologist, William Harvey Hospital, Kent, UK; Email: meerasabeka@gmail.com

Contribution of authors: Dr. Sabeka has contributed to clinical diagnosis, management, writing the manuscript. Dr. Mohammad Enayet Hussain diagnosed the case by electrophysiological study.

Conflict of interest: There is no conflict of interest relevant to this paper to disclose.

How to cite this article: Sabeka MM, Hussain ME. Dermatomyositis Presented with facial rash in a 40 Years old Lady in Bangladesh: A Case Report. J NatlInstNeurosci Bangladesh, 2019;5(2): 191-193

Copyright: (C2019. Sabeka and Hussain. Published by Journal of National Institute of Neurosciences Bangladesh. This article is published under the Creative Commons CC BY-NC License (https://creativecommons.org/licenses/by-nc/4.0/). This license permits use, distribution and reproduction in any medium, provided the original work is properly cited, and is not used for commercial purposes.

\section{Introduction}

Dermatomyositis is an inflammatory myopathy, involving proximal group of skeletal muscle with higher occurrence in middle aged female ${ }^{1}$. Diagnostic criteria include typical cutaneous features, progressive proximal symmetrical muscle weakness, elevated muscle enzymes and abnormal findings from muscle biopsy ${ }^{2}$.There is clinical and pathological overlap with connective tissue disease and malignancy occurs in $25 \%$ of cases ${ }^{3}$. Corticosteroids are the foundation of treatment and physical and occupational therapy should be started at the inception of diagnosis. Prognosis of the disease ought to be monitored by the improvement in the strength of the muscle and CPK levels.

\section{Case Presentation}

A forty year old ladywas admitted to a private hospital in the center of Dhaka city, with facial rash and generalized weakness. The symptoms started with fever which was thought to be viral or seasonal. Her febrile illness subsided with no medical intervention, however, weakness persisted and was gradually progressing. She was going downhill, being unable to move within a month of her febrile illness. She also had problem with swallowing and speaking with nasal voice. Dysphagia led to reduced intake. Later she developed generalized swelling (anasarca) which may be due to nutritional deficiency. Her vitals were normal. Hyper-pigmented maculopapular erythematous rash was seen on the forehead, nose, cheeks with upper eyelids edema and vitiligo involving front and back of the neck and lips. No Gottron papules or nail fold capillary abnormalities were seen. She had ankle oedema (pitting). She was less able to speak, with faint nasal voice, however, she was alert and could co-operate with full effort for physical examination. Her muscle power was zero; all deep tendon reflexes were normal with down-going plantar.

Her respiratory, cardiovascular and abdominal systems were normal. Battery of laboratory examinations revealed normal haemoglobin $(13.0 \mathrm{gm} / \mathrm{dL})$, Raised 


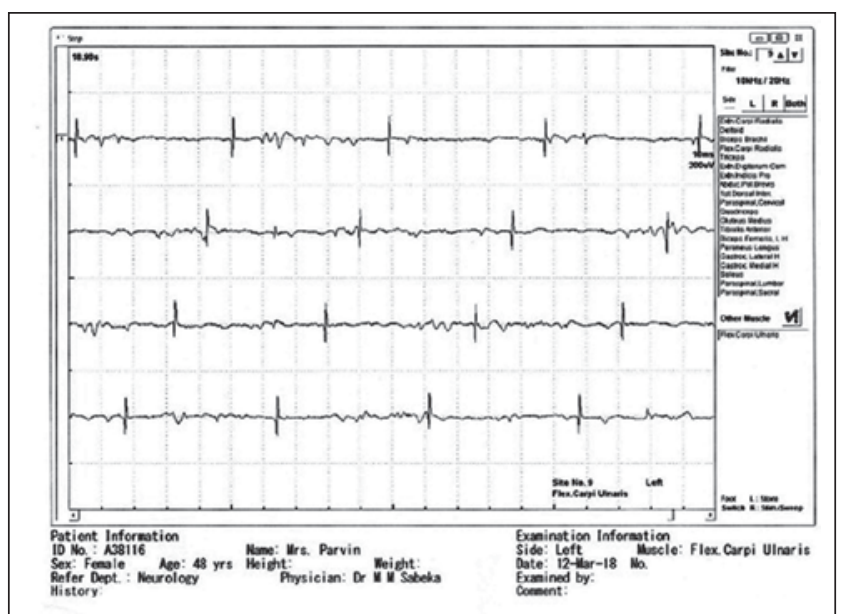

Figure I: Fibrillation

WBC count -16,500/cumm (Normal 4000$11000 /$ cumm), reduced platelets $-86,000 /$ cumm (Normal 1500000, -500000), raised ESR-75 mm/1 h. Urine analysis, renal function tests and blood glucose were within normal limits.

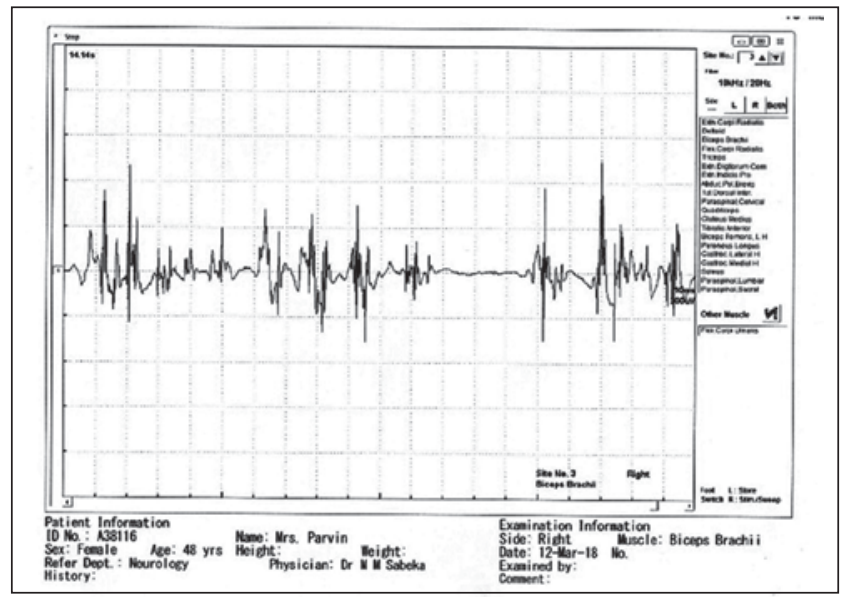

Figure II: Polyphasic, low amplitude MUPs

The liver function test showed raised ALT-218 IU/L (reference: 0-40 IU/L). Creatinine phosphokinase14,317 IU/L (reference: 24-171 IU/1), Serum albumin $12.3 \mathrm{gm} / \mathrm{L}$ (ref $34-50 \mathrm{gm} / \mathrm{L}$ ) and ultrasonography of abdomen showed ascites. TSH was $9.13 \mathrm{U} / \mathrm{L}$ (ref 0.47-5.01), normal FT4, with negative anti TPO and thyroglobulin antibody. Her RA, ANA, Anti dsDNA, anti SCL 70 were negative. Serum morning free cortisol was $23.50 \mathrm{microgm} / \mathrm{dL}$ (ref 1.7-19.4).Her ECG depicted sinus tachycardia with low voltage QRS complex. Chest $\mathrm{X}$-ray revealed bilateral pulmonary fibrosis.

Ultrasonography of breast showed features of congestion in right breast and multiple enlarged lymph node in the left axillary area. However, the FNAC for the axillary lymph nodes revealed no malignant cell. Motor NCS revealed reduced CMAP amplitude with

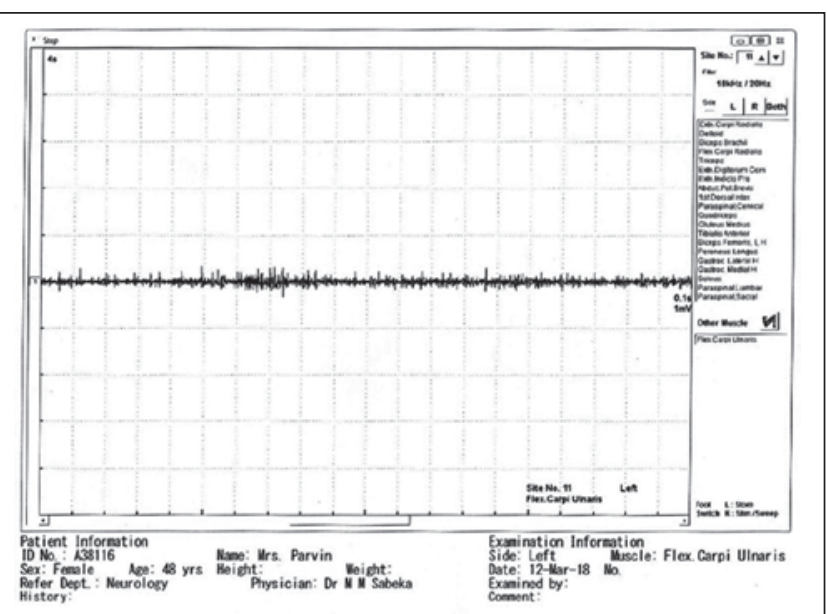

Figure III: Reduced recruitment

normal sensory NCS findings. EMG depicted profuse positive sharp waves and fibrillation potentials, with small polyphasic MUAPs (motor Unit Action Potentials) of brief duration along with early and full recruitment noted particularly in the proximal muscles. All these features pointing towards the clinical diagnosis of inflammatory myopathy. Considering the muscle weakness, facial rash, raised CPK and EMG findings a diagnosis of dermatomyositis was made. However, no clue could be identified to point the

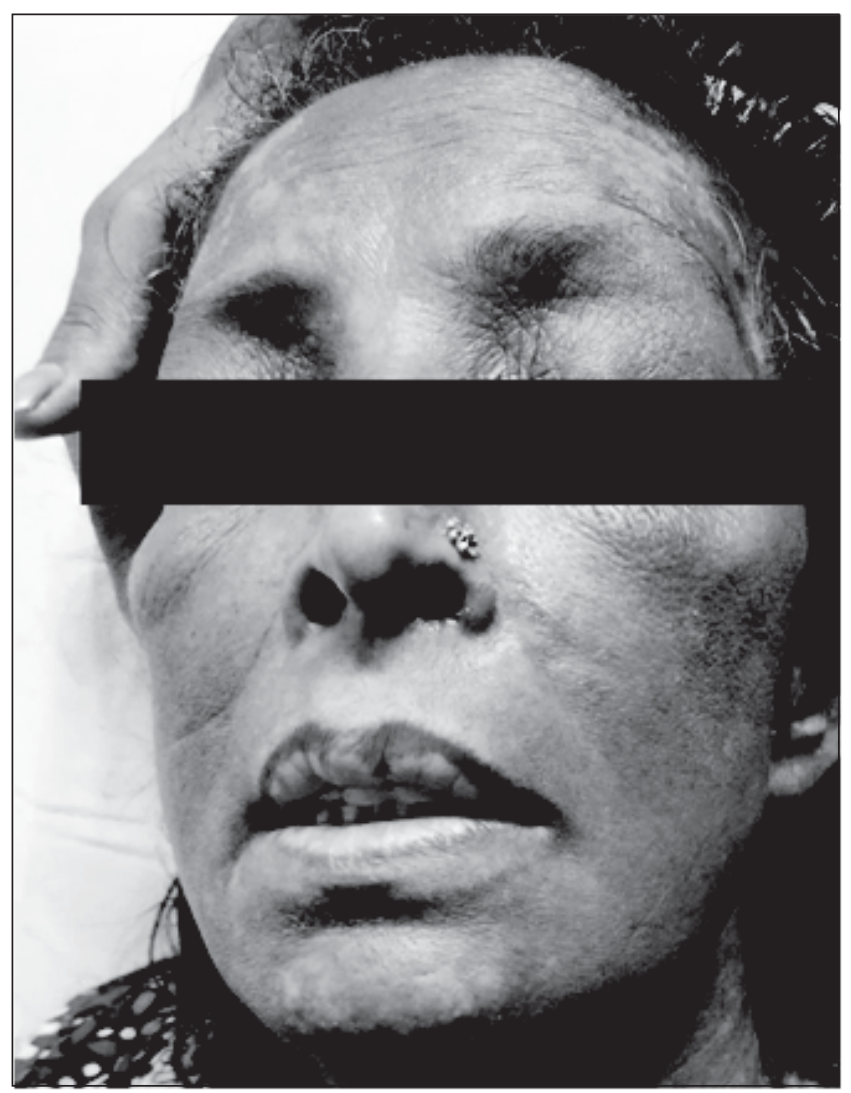

Figure IV: facial rash and vitiligo 
association for her condition, for example, connective tissue disease or malignancy. She was started on corticosteroid $1 \mathrm{mg} / \mathrm{kg} /$ day. Her condition was on the way to improvement.

\section{Discussion}

Dermatomyositis is an inflammatory myopathy characterized by cutaneous lesions. The incidence of $\mathrm{DM}$ is estimated at 1 to 10 per million in adults and at 1 to 3.2 per million in children ${ }^{4}$. The most commonly used diagnostic criteria for dermatomyositis are the Bohan and Peter criteria published in 1975: progressive proximal muscle weakness, elevated muscle enzymes, abnormal electromyogram, abnormal muscle biopsy, and classic cutaneous findings. Pathognomonic signs are heliotrope rash and Gottron papule 5 . For a definitive diagnosis of DM, the Bohan and Peter classification requires a characteristic rash plus 3 of the criteria of symmetrical, subacute, proximal muscle weakness, muscle biopsy abnormalities like necrosis, regeneration, fiber size variation, inflammation, and atrophy and muscle enzyme level elevation like CK, aldolase, AST, ALT, and LDH as well as the electromyographic abnormalities like triad of short, small, polyphasic motor units; insertional irritability, positive sharp waves, fibrillations and bizarre, high-frequency repetitive discharges; furthermore, rash typical of DM like heliotrope rash or Gottron papules.

There is no specific diagnostic test or universally accepted diagnostic criteria for dermatomyositis. On the other hand, the initial presentation may be vague, that renders early diagnosis difficult. The muscle biopsy is central to establishing the diagnosis, but selecting the appropriate site for biopsy may be difficult. Muscles with end-stage disease should be avoided because the pathological findings often are nonspecific, showing only atrophy and maybe inflammation.

Dermatomyositis is known to be associated with connective tissue disease, interstitial lung disease, cardiac disease and malignancy. Pulmonary complications include interstitial lung disease (ILD), aspiration pneumonia, and hypoventilation resulting from respiratory muscle weakness ${ }^{3}$. Cardiac Manifestations include myocarditis (rarely leading to heart failure), pericarditis, valvular disease, and rhythm disturbances ${ }^{3}$. Early diagnosis is necessary not only for prompt treatment, but also for screening and monitoring for malignancy.
Several lines of evidence showed the link between DM and neoplastic disease. The first report of dermatomyositis associated with stomach cancer, by Stertz, comes from 1916. In the same time, Kankeleithas reported that DM associated with breast cancer. Presumably, it is the result of immune reaction against antigens common for muscle and neoplastic cells or some paraneoplastic syndrome underlying mechanism ${ }^{4}$.

Physical therapy and occupational therapy should be started at diagnosis. Active disease should not prevent patient-specific graded exercise ${ }^{6}$. Corticosteroids are the foundation of treatment. Oral prednisone usually is initiated at $1 \mathrm{mg} / \mathrm{kg} / \mathrm{d}$ in divided doses to a maximum of $80 \mathrm{mg} / \mathrm{d}$ and continued until the CK level improves (often after 4 weeks). Low-dose prednisone usually is continued to complete a year of therapy. Patients should be evaluated for response to therapy monthly, with measurement of muscle enzyme levels, manual muscle testing, and assessment of functional status?

\section{Conclusion}

Dermatomyositis is an inflammatory myopathy, which may or may not be secondary to any malignancy. There is no single diagnostic tests, no universal diagnostic criteria either. In this case, muscle biopsy was not possible due to reduced muscle bulk and deteriorated physical condition of the patient. High index of suspicion is mandatory for early diagnosis and management. Drug treatment should be judicial, ethical and according to protocol, where appropriate. Life-long monitoring is life-saving.

\section{References}

1. KalyanM, Kanitkar SA, Gaikwad AN, Kumar H. Dermatomyositis, a case report. Journal of Mahatma Gandhi Institute of Medical Sciences 2016;21(1):53-55

2. Bohan A, Peter JB.Polymyositis and dermatomyositis (second of two parts). N Engl J Med 1975;292:403-7

3. Velderman PN, Bruch LA, Lawry LA. Identifying and Managing Dermatomyositis: A Case Report and Review. J Musculoskel Med. 2008;25:415-420

4. GrzegorzPrzybylski, AgnieszkaJarzemska, JarosławCzerniak et al. A case report of a patient with dermatomyositis as a prodromal sign of lung cancer. Pol Arch Med Wewn 2008; 118(3): 143-147 5. Judy Truong, Christofer $P$ et al. Looking for signs: A dermatomyositis case report.Journal of the American Academy of Physician Assistants: December 2015 - Volume 28 - Issue 12 - p 1 6. Coyle KM, Plotz PH, Gourley MF. Why isn't my myositis patient getting better? The Rheumatologist. 2008;2:18-19

7. Oddis CV. Idiopathic inflammatory myopathy: management and prognosis. Rheum Dis Clin North Am. 2002;28:979-1001 